\title{
QoE Evaluation Model Based on Video Content Complexity
}

\author{
Nan Wang
}

School of Software Engineering, Beijing University of Technology, Beijing 100000, China. 12810220@qq.com

Keywords: QoE, Model, Video content

\begin{abstract}
With the mobile communication technology developing rapidly, wireless bandwidth can be used more and more, which will make high-speed video services occupy most of the wireless communication traffic. This paper presents a QoE evaluation model based on video content, combined with video jitters and packet loss rate. The experimental results show that the evaluation results of the model can reflect the video QoE better and have good performance.
\end{abstract}

\section{INTRODUCTION}

In the process of network technology developing rapidly, real-time data such as voice and video in the network are in large scale[1]. However, due to the limited bandwidth of the network, the video needs to be compressed before transmission. In the process of transmission and other issues, which led to the user finally received the video data, and the original video data there are some differences. The degree of this video distortion will have a significant impact on the Quality of Experience (QoE)[2].

At present, the existing video evaluation standard is mainly through the difference between the source video and received video's parameters to get the video distortion, or combined with the transmission process of network services parameters to establish the QoE evaluation model[3]. However, this evaluation standard ignores the influence of video content on the subjective feelings of users. This paper consider the characteristics of video content and the quality of service parameters of transmission network, and establishes a QoE evaluation standard based on video content . In the test of mathematical models it also considers the user's subjective feelings.

\section{ORGANIZATION OF THE TEXT}

\section{The effect of video jitters and packet loss on QoE}

The jitters in the network makes the video stream receiving time not constant, usually if the video stream packet jitters time exceeds a certain threshold range decoder, it will discard this packet, which directly affect the video stream in the terminal display[4]. In this section, the QoE values of video transmission under different jitters conditions are obtained experimentally. The delay is set to $120 \mathrm{~ms}$ during the experiment. The experimental results are shown in Figure 1. At the same time, the experimental results shows when video objects and background changes violently, the user experience value will be more sensitive to jitters. 


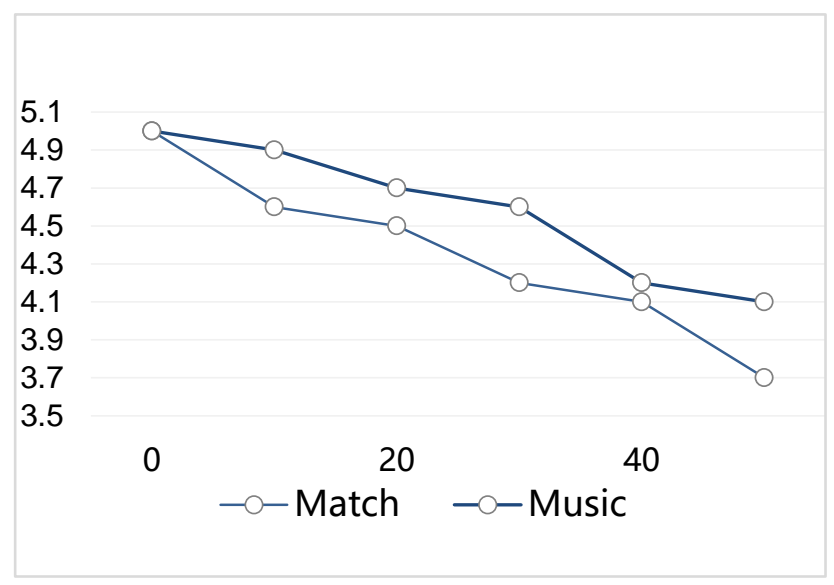

FIGURE 1. Effect of jitters on the video quality.

Packet loss is an important factor on affecting the quality of network video[5]. As the transmission of video files in the network is usually packaged into a fixed size of the packet, different groups contain different coding types of fragment information. Once the packet loss occurs, the initial damage occurs. And this packet loss will also affect the encoding and decoding of the relevant frame[6]. In this paper, we study the quality of user experience under different packet loss rates. The experimental results are shown in Figure 2.

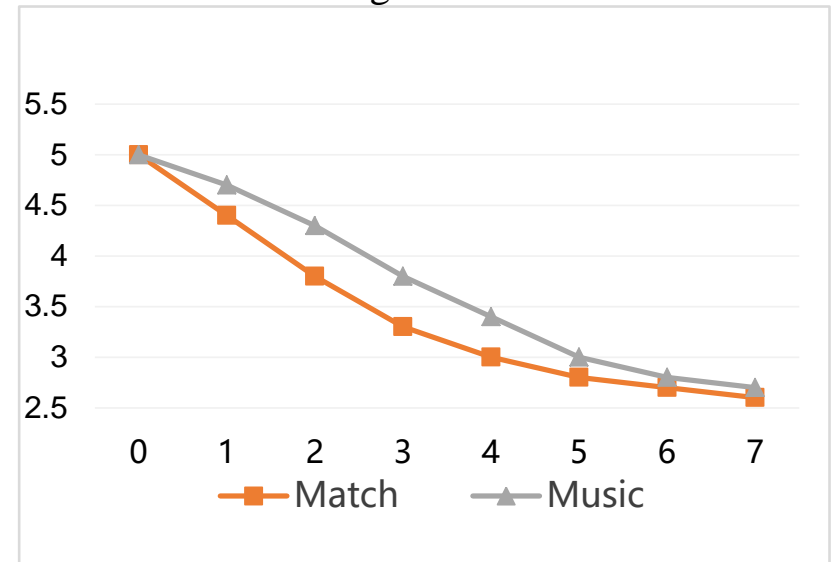

FIGURE 2. Effect of packet loss on the video quality (packet loss rate (\%))

\section{Research on the Relationship between video content features and QoE The impact of video content on the QoE}

The quality of the network video is mainly affected by the compression of the video in the coding process, the packet loss during transmission[7], and the influence of the network jitters. As for the content of different video sequences, which are in the same encoding and transmission conditions, the quality will be very different[8]. The relationship between the bit rate of these four experimental video sequences and their MOS values is as follows. It can be seen from Figure 3 that the MOS of the four video sequences increases with the increase of the coding bit rate and eventually stabilizes, but they increase amplitude is different, and their MOS differences are large at the same coding bit rate, which is due to the different content characteristics of the video sequence. Through the above analysis, only considering the video encoding bit rate, packet loss rate, other basic video parameters and network quality of service parameters will not be able to accurately evaluate the video QoE[9]. Therefore, this paper takes into account the above parameters, combined with the video content features to evaluate the video QoE. 


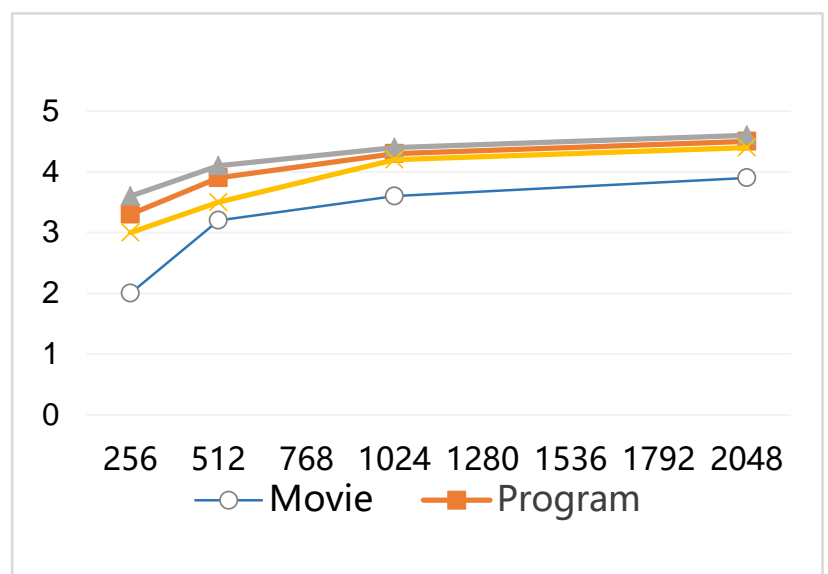

FIGURE3. Relationship between the MOS of the video sequence with different content

\section{The definition of video content complexity} characteristics and coding rate

In this paper, the content complexity factor is constructed by using the relevant parameters of motion vector and intra-coding macro-block. Figure 4 and Figure 5 show the case where the motion vector of the experimental video sequence and the correlation parameter of the intra-coding macroblock are changed with the coding rate. In Figure 4, $\mathrm{m}$ is the average of the motion vectors of each macro-block of the whole sequence. I in Figure $5 \mathrm{i}$ is the average of the number of intra-coding macro-blocks in each frame of the entire video sequence. By observing the trend of the above two parameters, it can be seen that the correlation between the size of the motion vector and the motion characteristics of the video can be regarded as the main determinant of the content complexity of the video sequence.
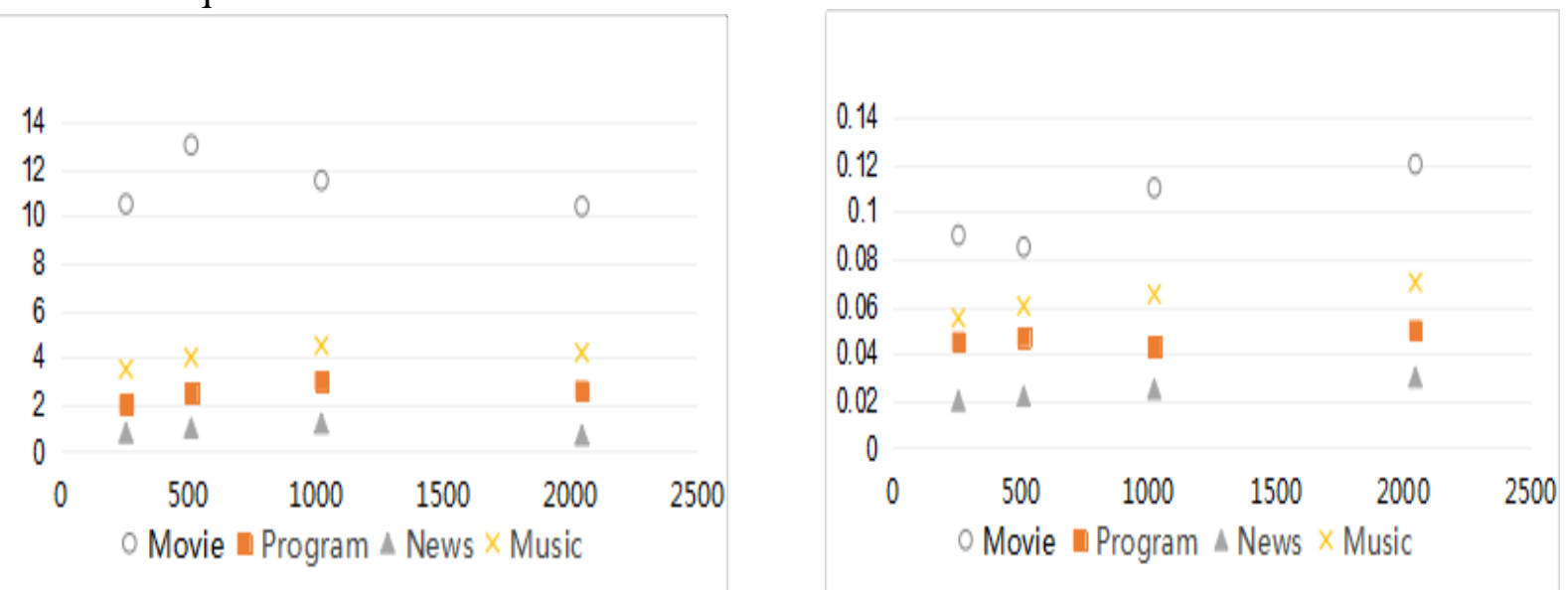

FIGURE 4. The relationship between $\mathrm{m}$ and the coding rate between $i$ and the coding rate

FIGURE 5. The relationship

So we finally define the content complexity factor as:

$$
\begin{aligned}
& f=f_{1}(m)+f_{2}(i, r) \\
& f_{1}(m)=m^{2} \\
& f_{2}(i, r)=\frac{a \times i}{\log (r)} \\
& f=m^{2}+\frac{a \times i}{\log (r)}
\end{aligned}
$$

Where $f$ is the content complexity factor, $m$ is the average of the motion vectors, $a$ is the adjustment coefficient, $i$ is the average of the total macro-block ratio occupied by the intra-coding macro-block, $r$ is the coding rate, in $\mathrm{kb} / \mathrm{s}$. The more complex the content complexity factor will be, the content complexity factor can better reflect the motion characteristics of the video sequence.

The QoE evaluation model based on the video content characteristics

Two main factors that affect QoE: the coding distortion[10] caused by the encoding before transmission and the transmission distortion[11] caused by packet loss and network jitters during 
transmission. In this paper, the coding distortion model and the transmission distortion model are established and integrated as the final video QoE evaluation model.

\subsection{Coding distortion model}

Through the analysis and comparison of the existing models, the model formula of the international standard G.1070 model[12] is in line with the actual correspondence between the coding rate and the quality of the video after coding distortion:

$$
\begin{aligned}
& I_{c}=v_{1} \times\left(1-\frac{1}{1+\left(\frac{r}{v_{2}}\right)^{v_{3}}}\right) \\
& Q_{c}=1+I_{c}
\end{aligned}
$$

In the formula, $I_{c}$ is the video quality of the video after encoding distortion, $v_{1}, v_{2}, v_{3}$ are the undetermined coefficient, $Q_{c}$ is the quality of the user's experience after encoding distortion.

\section{Transmission distortion model}

The transmission distortion model is usually expressed as an exponential[14]:

$$
Q_{t}=1+I_{c} \times \exp \left(\frac{p l f+j i t}{v_{4}}\right)
$$

Where $I_{c}$ is the quality of the video in the coding distortion, plf is the packet loss rate, jit is the network jitters, $Q_{c}$ is to be determined for the quality of the user after the distortion of transmission. Then get the transmission distortion model:

$$
Q_{t}=1+I_{c} \times \exp \left(\frac{p l f+j i t}{v_{41} \times\left(m^{2}+\frac{a \times i}{\log (r)}\right)+v_{42}}\right)
$$

Based on the integrated coding distortion model and the transmission distortion model, the final QoE model of the video sequence can be obtained as follows:

$$
Q_{t}=1+v_{1} \times\left(1-\frac{1}{1+\left(\frac{r}{v_{2}}\right)^{v_{3}}}\right) \times \exp \left(\frac{p l f+j i t}{v_{4}}\right)
$$

At this point, this paper establishes a QoE evaluation model based on video content.

\section{Experimental results and performance analysis}

Experiments selected four video sequences, the testers conduct subjective quality assessment by QSCQS.

This paper in the original assessment method based on the improvement, requiring the tester to watch the original video and test video, the tester scores the test video by observing the difference between the two according to the Five-Point scale in the ITU-R Quality and Damage Scale. Finally, the average of the post-screening score data is taken as the MOS score of the video sequence.

In order to verify the accuracy of the QoE evaluation model, this paper calculates the objective evaluation scores of all the video sequences using the model and compares the MOS scores obtained directly from the subjective test. The performance parameters in Table 1 show that the model established in this paper can accurately assess the QoE of different content video, indicating that the QoE evaluation model has a good performance. 
TABLE 1. Pearson correlation coefficient and root mean square error

\begin{tabular}{ccc}
\hline Score Level & PC & RMSE \\
\hline Movie & 0.93 & 0.21 \\
Program & 0.97 & 0.24 \\
News & 0.95 & 0.35 \\
Music & 0.98 & 0.32 \\
\hline
\end{tabular}

\section{CONCLUSION}

This paper presents a QoE evaluation model based on the characteristics of video content. The model not only takes into account the distortion caused by the video during the coding process, the loss of transmission caused by packet loss and network jitters during the transmission process, it also studies the video content influences. The experimental results show that the objective mass fraction predicted by the model is in good agreement with the subjective mass fraction. Due to its excellent performance, the model can accurately evaluate the quality of the video and can be applied to real-time monitoring and evaluation of the network video service.

\section{REFERENCES}

[1] Rappaport T S, Sun S, Mayzus R, et al. Millimeter Wave Mobile Communications for 5G Cellular: It Will Work![J]. Access IEEE, 2013, 1(1):335-349.

[2] Jain. Quality of Experience[C]// International Conference on Computing, Management and Telecommunications. IEEE, 2013:205-257.

[3] Yang F, Song J, Wan S, et al. Content-Adaptive Packet-Layer Model for Quality Assessment of Networked Video Services[J]. IEEE Journal of Selected Topics in Signal Processing, 2012, 6(6):672-683.

[4] Wang Q, Liu Y, Yu D, et al. Method, apparatus, and system for adjusting multimedia encoding rate: US, US8467409[P]. 2013.

[5] Wu H R, Rao K R, Kassim A A. Digital Video Image Quality and Perceptual Coding[J]. Journal of Electronic Imaging, 2005, 16(16):039901.

[6] Juan, Lin, Yiwen, et al. Packet-Layer Quality Assessment for Networked Video[J]. International Journal of Computers Communications \& Control, 2014, 7(3):565-573.

[7] Van Der L R. Recovery From Packet Loss During Transmission of Compressed Video Streams:, US20140269917[P]. 2014.

[8] Paudyal P, Battisti F, Carli M. Impact of video content and transmission impairments on quality of experience[J]. Multimedia Tools \& Applications, 2016, 75(23):16461-16485.

[9] Raake A, Garcia M N, Moller S, et al. T-V-model: Parameter-based prediction of IPTV quality[C]// IEEE International Conference on Acoustics, Speech and Signal Processing. IEEE, 2008:1149-1152.

[10] Xue Z, Shen S M, Foo T W, et al. Coding distortion removal method, video encoding method, video decoding method, and apparatus and program for the same: US, US 7742531 B2[P]. 2010.

[11] He Z, Xiong H. Transmission Distortion Analysis for Real-Time Video Encoding and Streaming Over Wireless Networks[J]. IEEE Transactions on Circuits \& Systems for Video Technology, 2006, 16(9):1051-1062.

[12] Belmudez B, MöLler S. Extension of the G.1070 video quality function for the MPEG2 video codec[C]// Second International Workshop on Quality of Multimedia Experience. IEEE, 2010:7-10.

[13] Furuta S, Hongo N. Distortion compensation amplifying apparatus: US, US7514996[P]. 2009. 